\title{
High-Frequency Acoustic Droplet Vaporization is Initiated by Resonance
}

\author{
Guillaume Lajoinie $\odot,{ }^{*}$ Tim Segers $\odot,{ }^{*}$ and Michel Versluis ${ }^{\dagger}$ \\ Physics of Fluids Group, MESA+ Institute for Nanotechnology, Technical Medical (TechMed) Center, University of Twente, \\ P.O. Box 217, 7500 AE Enschede, Netherlands
}

(Received 30 June 2020; revised 2 October 2020; accepted 1 December 2020; published 19 January 2021)

\begin{abstract}
Vaporization of low-boiling point droplets has numerous applications in combustion, process engineering, and in recent years, in clinical medicine. However, the physical mechanisms governing the phase conversion are only partly explained. Here, we show that an acoustic resonance can arise from the large speed of sound mismatch between a perfluorocarbon microdroplet and its surroundings. The fundamental resonance mode obeys a unique relationship $k R \sim 0.65$ between droplet size and driving frequency that leads to a threefold pressure amplification inside the droplet. Classical nucleation theory shows that this pressure amplification increases the nucleation rate by several orders of magnitude. These findings are confirmed by high-speed imaging performed at a timescale of $10 \mathrm{~ns}$. The optical recordings demonstrate that droplets exposed to intense acoustic waves generated by interdigital transducers nucleate only if they match the theoretical resonance size.
\end{abstract}

DOI: 10.1103/PhysRevLett.126.034501

Vaporization of low-boiling point droplets is omnipresent in today's society with applications in renewable energy and energy storage [1], combustion [2], intumescent fire-protective coatings [3], and recently in clinical medicine [4,5]. Deterministic vaporization can be initiated by heat or negative pressure, and by combinations thereof [6], which allows droplet vaporization to be triggered by laser light [7,8], ultrasound [9], neutrons [10], and protons [11]. Ultrasound-triggered phase change of superheated nanoand microdroplets is known as acoustic droplet vaporization (ADV) [12-15]. ADV is of great interest for medicine since submicrometer-sized surfactant-stabilized droplets, or nanodroplets, have been shown to be able to extravasate leaky tumor vasculature thereby passively accumulating within the tumor $[16,17]$. Upon phase change, the formed bubbles can perform therapeutic action such as local drug delivery and sonoporation [18-20].

The interaction of ultrasound with a low-boiling point droplet has been subject to extensive study aiming at understanding the underlying physical mechanisms driving nucleation. Experimentally, it has been found that a prominent peak negative pressure (PNP) nucleation threshold exists, above which the nucleation probability increases with the acoustic pressure amplitude and that this threshold, counterintuitively, lowers with an increase in ultrasound frequency and with a decrease in ambient pressure $[9,12,21-30]$. These observations are in line with what is predicted from both classical nucleation theory [31] and superharmonic focusing [23,32], and by the combination of the two $[33,34]$. Superharmonic focusing results from the focusing of higher harmonics with wavelengths on the order of the droplet diameter that are generated through nonlinear propagation of the transmitted ultrasound wave
[32]. However, the required nonlinear propagation in tissue is dramatically lower from that in water due to the 2 orders of magnitude lower ratio of acoustic nonlinearity to attenuation, or Gol'dberg number [35]. This severely limits the effectivity of ADV by superharmonic focusing in vivo. In this Letter, a physical ADV nucleation mechanism based on an acoustic resonance of the droplet is presented that has been overlooked until now. The theoretical resonance behavior is experimentally validated and its role in lowering the vaporization threshold is elucidated using classical nucleation theory. Resonant ADV offers an efficient approach for in vivo applications and adds to our fundamental understanding of acoustic droplet vaporization.

Here we will give the main results of the derivation; all details can be found in the Supplemental Material SI.1 [36]. The system consists of a perfluoropentane (PFP) droplet (medium 1) immersed in water (medium 0) considered to be of infinite size. We assume a purely spherical geometry. To calculate the resonance behavior we couple the pressure on the inside of the droplet interface $p\left(R_{\text {in }}, t\right)$ to the external acoustic driving pressure $p_{A}(t)$. The droplet is assumed to be small compared to the wavelength in water $\lambda_{0}$ and the acoustic pressure can be considered homogeneous around the droplet. Integration of the momentum equation in water then gives

$$
p\left(R_{\text {out }}, t\right)=\left[p_{\text {atm }}+p_{A}(t)\right]+\rho_{0}\left(R \ddot{R}+\frac{3}{2} \dot{R}^{2}\right),
$$

with $p\left(R_{\text {out }}, t\right)$ the pressure on the outside of the droplet interface. $p_{\text {atm }}$ is the atmospheric pressure, $R(t)$ is the droplet radius, and its overdots represent the interface 
velocity and acceleration, respectively. The pressure jump across the interface is expressed using the normal stress balance:

$$
p\left(R_{\mathrm{in}}, t\right)-p\left(R_{\mathrm{out}}, t\right)=4\left(\mu_{0}-\mu_{1}\right) \frac{\dot{R}}{R}+\frac{2 \sigma}{R},
$$

with $\mu_{i}$ the viscosity of medium $i$. While in the analogous derivation of the Rayleigh-Plesset equation for bubbles the equation is closed by the highly compressible and uniform gas pressure [37], here we need to evaluate the complete acoustic pressure distribution within the droplet. A classical acoustic derivation of the particle velocity $v$ leads to the well-known spherical Bessel equation:

$$
x^{2} \frac{\partial^{2} v}{\partial x^{2}}+2 x \frac{\partial v}{\partial x}+\left(x^{2}-2\right) v=0 .
$$

Here, $x=k_{1} r$, with $k_{1}=\omega / c_{1}$ the wave number, $c_{1}$ the speed of sound in the droplet, and $r$ the radial coordinate. Avoiding the unphysical divergence at $r=0$, the solution to Eq. (3) is a spherical Bessel function of the first kind:

$$
v(r, t)=f(t) j_{1}(x)=f(t) \frac{\sin x-x \cos x}{x^{2}} .
$$

Writing $X=k_{1} R$, the boundary condition at the droplet interface $v(R, t)=\dot{R}$ yields

$$
v(r, t)=\dot{R} \frac{j_{1}(x)}{j_{1}(X)} .
$$

The pressure distribution is then found by inserting Eq. (5) in the mass conservation and compressibility equation and using the Bessel function recurrence relation $j_{1}^{\prime}(x)=j_{0}(x)-2 j_{1}(x) / x$ :

$$
\frac{\partial p}{\partial t}=-\frac{1}{\beta_{1}}\left(\frac{\partial v}{\partial r}+2 \frac{v}{r}\right)=-\rho_{1} k_{1} c_{1}^{2} \dot{R} \frac{j_{0}(x)}{j_{1}(X)},
$$

where $\beta_{1}=1 / \rho_{1} c_{1}^{2}$ is the compressibility of the droplet. Note that, owing to the $j_{0}(x)=\sin x / x$ sinc term, the pressure amplitude will always be maximum in the center of the droplet. Integrating Eq. (6) for small oscillation amplitudes $\left[R=R_{0}(1+\epsilon), \epsilon \ll 1\right]$ with $R_{0}$ the resting radius and $X_{0}=k_{1} R_{0}$, with the initial boundary condition $p(r, 0)=p_{\text {atm }}+2 \sigma / R_{0}$ at $t=0$ and evaluating in $r=R$ yields

$p\left(R_{\mathrm{in}}, t\right)=\left(p_{\mathrm{atm}}+2 \sigma / R_{0}\right)-\rho_{1} k_{1} c_{1}^{2} \frac{j_{0}(X)}{j_{1}\left(X_{0}\right)}\left(R-R_{0}\right)$.

Note that this approximation only holds when $j_{1}\left(X_{0}\right)$ is not near its zero crossing. Combining Eqs. (1), (2), and (7) now gives the droplet dynamics equation

$$
\begin{aligned}
\rho_{0}\left(R \ddot{R}+\frac{3}{2} \dot{R}^{2}\right)= & -\rho_{1} k_{1} c_{1}^{2} \frac{j_{0}(X)}{j_{1}\left(X_{0}\right)} R\left(1-\frac{R_{0}}{R}+\frac{\dot{R}}{c_{0}}\right) \\
& -4\left(\mu_{0}-\mu_{1}\right) \frac{\dot{R}}{R}-2 \sigma\left(\frac{1}{R}-\frac{1}{R_{0}}\right)-p_{A}(t) .
\end{aligned}
$$

The reradiated pressure scattered by the droplet induces a compression of the surrounding medium. The effect of acoustic reradiation on the droplet dynamics can be expressed by an additional pressure term $\left(R / c_{0}\right)(\partial p / \partial t)$ at $r=R$ [38], effectively adding a damping term to the set of equations. The above equations can also be extended to include the acoustic interaction with a rigid wall through the addition of a pressure term $\rho_{0}(\partial / \partial t)\left(R^{2} \dot{R} / 2 d\right)$ representing the reflected scattering, with $d$ the distance to the wall [39]. For a droplet at the wall, $d=R$, and the left-hand side of Eq. (8) takes the form $\rho_{0}\left(\frac{3}{2} R \ddot{R}+2 \dot{R}^{2}\right)$, see Supplemental Material SI.2 [36]. Equation (8) can be solved numerically to obtain the resonance behavior of the system. The pressure in the center of the droplet can be obtained by reevaluating Eq. (6) in $r=0$ instead of $r=R$ :

$$
p_{\text {drop }}=\left(p_{\text {atm }}+2 \sigma / R_{0}\right)-\rho_{1} k_{1} c_{1}^{2} \frac{\left(R-R_{0}\right)}{j_{1}\left(X_{0}\right)} .
$$

Linearization of Eq. (8) gives a relation between the angular eigenfrequency of the droplet $\omega_{0}$ and its resting radius:

$$
\rho_{0}\left(\omega_{0} R_{0}\right)^{2}=\frac{\rho_{1} \omega_{0} R_{0} c_{1}}{\frac{c_{1}}{\omega_{0} R_{0}}-\cot \left(\frac{\omega_{0} R_{0}}{c_{1}}\right)}+\frac{2 \sigma}{R_{0}} .
$$

Using a first-order expansion $\cot (z) \simeq 1 / z-z / 3+\mathcal{O}\left(z^{3}\right)$ and by neglecting the interfacial tension term, Eq. (10) reduces to a simple classical form:

$$
f_{0} \simeq \frac{1}{2 \pi R_{0}} \sqrt{\frac{3 \rho_{1} c_{1}^{2}}{\rho_{0}}},
$$

with $f_{0}=\omega_{0} / 2 \pi$ the eigenfrequency of the droplet. The same approach leads to an expression for the damping of the system in canonical form:

$$
\delta=\frac{\omega_{0} R_{0}}{2 c_{0}}+\frac{2\left(\mu_{0}-\mu_{1}\right)}{\rho_{0} \omega_{0} R_{0}^{2}} .
$$

As a result of the negligible contribution of viscous damping, which is 3 orders of magnitude smaller than the first term in Eq. (12), this then further reduces to

$$
\delta \simeq \frac{c_{1}}{c_{0}} \sqrt{\frac{3 \rho_{1}}{4 \rho_{0}}} .
$$



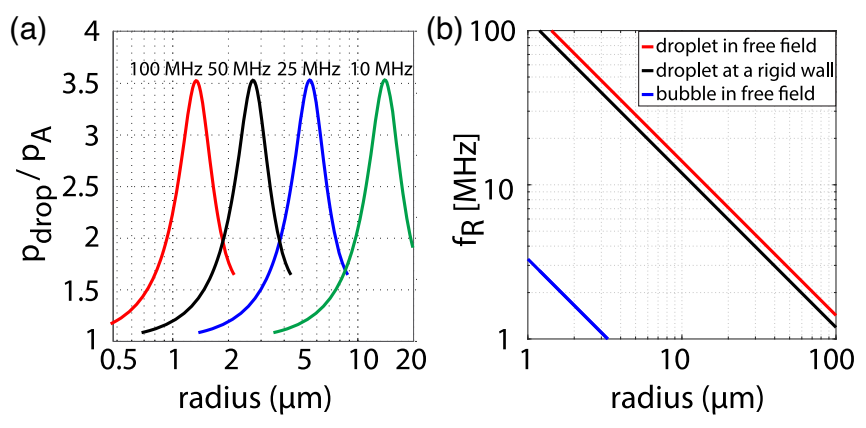

FIG. 1. (a) Calculated theoretical resonance curves for different driving frequencies. (b) Calculated resonance frequency $f_{R}$ as a function of size: droplet in free-field (red), droplet at a rigid wall (black), and bubble in free field (blue).

For a droplet at the wall the linearization simply adds a prefactor $\sqrt{2 / 3}$ to both Eqs. (11) and (13). Equation (13) also shows that the resonance arises from a speed of sound mismatch between the droplet and its surrounding medium, i.e., when the speed of sound ratio goes to 1 , the system becomes overdamped, without a resonance effect, while the largest speed of sound mismatch produces the strongest resonance.

The resonance curves, solutions of Eq. (8) and computing the maximum pressure in the drop using Eq. (9) for frequencies of $10,25,50$, and $100 \mathrm{MHz}$ were calculated using the ODE45 solver in MATLAB and are displayed in Fig. 1(a). The physical parameters for PFP and water that were used for the calculations are listed in Supplemental Material SI.3 [36] and were extracted from Refs. [40-42]. The resonance frequency $f_{R}$ is plotted against the droplet size in Fig. 1(b) for a droplet in free field and for a droplet against a rigid wall. Two direct results from these plots are that (i), unlike superharmonic focusing, the acoustic resonance strength has very little dependency on droplet size (see also Supplemental Material SI.4 [36]) and (ii), the resonance is expected to have a high quality factor and a frequency about 50 times higher than that of free gas bubbles, see the corresponding Minnaert bubble resonance frequency $f_{M}=3.3 \mu \mathrm{mMHz} / R_{0}$ plotted in Fig. 1(b) [37].

Owing to the high eigenfrequencies expected, the response of PFP droplets was measured experimentally at frequencies of 19.6 and $45.4 \mathrm{MHz}$. The droplets were resting on the piezoelectric substrate on which surface acoustic waves (SAW) were generated using an interdigitized transducer (IDT); see Fig. 2(a). In contact with water, the SAW generates a longitudinal bulk acoustic wave at the Rayleigh angle $\theta_{R} \approx 23^{\circ}$ [43-45], see Fig. 2(b). The use of a SAW device prevents any nonlinear propagation in the bulk of the medium, leaving a purely sinusoidal excitation. Straight electrode IDTs with a single aluminum electrode pair per wavelength (60 pairs, thickness of $750 \mathrm{~nm}$, aperture of $1 \mathrm{~cm}$ ) were fabricated on a $128^{\circ}$ rotated $Y$-cut $\mathrm{X}$-propagating lithium niobate $\left(\mathrm{LiNbO}_{3}\right.$, Roditi, United Kingdom) wafer using standard soft lithography
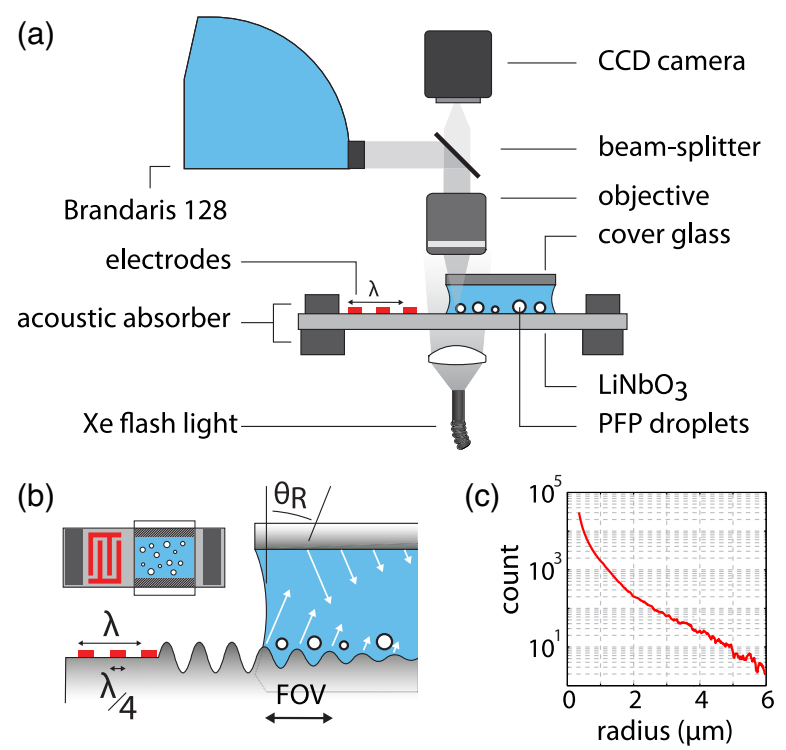

FIG. 2. (a) Schematic of the experimental setup. The vaporization of PFP droplets is imaged using the Brandaris 128 ultrahigh-speed camera. (b) A SAW device generates a longitudinal pressure wave in the fluid at the Rayleigh angle $\theta_{R}$. (c) Typical size distribution of the droplets used in this study.

techniques. The IDTs were actuated by a 50-cycle sinusoidal ultrasound pulse generated by a waveform generator (model 8026, Tabor Electronics) connected to a $50 \mathrm{~dB}$ linear power amplifier (350L, E\&I). A sound absorbing silicone rubber (PDMS, Dow Corning) was placed both below and above the end of the piezoelectric substrate to reduce acoustic reflections.

A perfluoropentane (PFP) droplet emulsion was prepared as in Ref. [15]. Its size distribution was measured using a Coulter counter; see Fig. 2(c). The suspension was loaded by capillary suction in a chamber that was approximately $100 \mu \mathrm{m}$ in height. The chamber was located directly above the piezoelectric substrate, open at the front end to allow for a direct coupling between the SAW and the liquid, and closed above using a microscope cover slip (24 mm length); see details in Fig. 2(b).

Droplet vaporization was imaged using an inverted microscope (Olympus BX-FM) equipped with a $20 \times$ magnification objective (Olympus SLMPlan N) coupled to the Brandaris 128 ultra high-speed camera [46,47] operated at $15 \times 10^{6}$ frames/ sec (Mfps) to record the time and location of droplet nucleation. The imaging resolution was $0.29 \mu \mathrm{m}$ per pixel. The field of view (FOV) was positioned close to the meniscus of the liquid, at the front end of the chamber, to minimize interference caused by acoustic reflections from the top of the chamber. Three successive high-speed recordings of 128 frames each were acquired at an interval of $100 \mathrm{~ms}$. Droplets were vaporized during the second recording and the first frames of the third recording were used to image the bubbles formed. The high-speed imaging frames were processed with an 
(a)

(b)

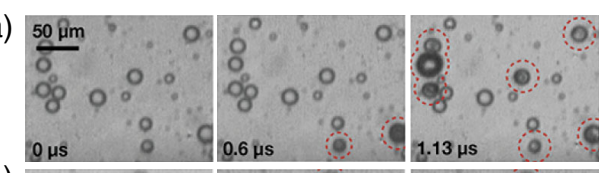

)

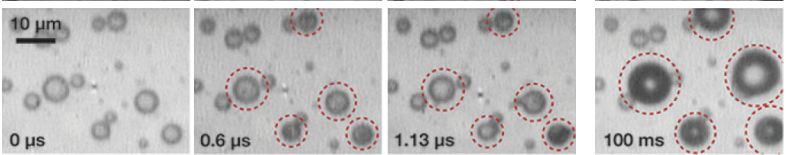

(c)
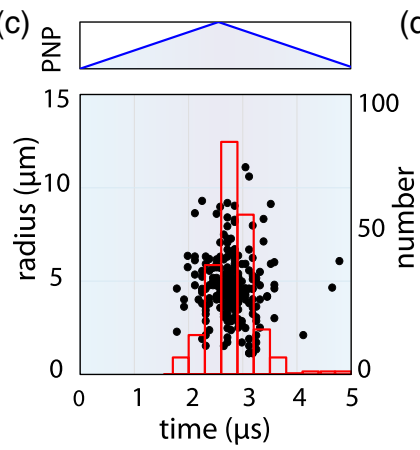

(d) $\stackrel{\mathrm{z}}{\mathrm{a}}$

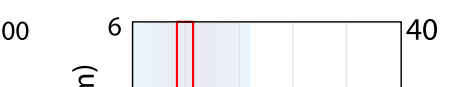

כิ

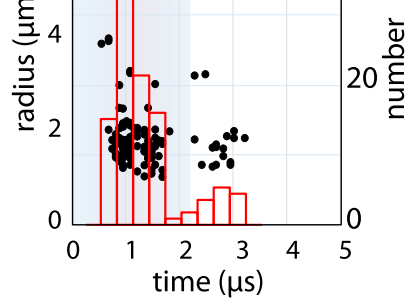

FIG. 3. Vaporization of PFP droplets driven at 19.6 (a) and 45.4 MHz (b) imaged at $15 \mathrm{Mfps}$. The nucleated droplets are marked by the red dotted circles. Stable bubbles are formed from all nucleated droplets as can be observed from the image captured $100 \mathrm{~ms}$ later. Number of nucleation events over time and the corresponding radius of the droplet at a driving frequency of 19.6 (c) and $45.4 \mathrm{MHz}(\mathrm{d})$.

automated image analysis procedure programmed in MATLAB (The MathWorks, Natick, MA). All experiments were performed at $20^{\circ} \mathrm{C}$.

Figure 3 shows an image sequence of the vaporization of PFP droplets driven at frequencies of 19.6 (a) and $45.4 \mathrm{MHz}$ (b). The droplets that underwent nucleation are marked by the red-dotted circles. At $19.6 \mathrm{MHz}$, the nucleated bubbles grow rapidly due to rectified heat transfer under acoustic forcing [48]. The bubble size subsequently decreased within microseconds after the ultrasound driving was stopped. ADV at $45.4 \mathrm{MHz}$ is less violent than at $19.6 \mathrm{MHz}$. In particular, the bubbles that nucleated inside the droplets were much smaller at 45.4 MHz. Note that every nucleation site produced a stable bubble at both driving frequencies, as can be observed from the images captured after $100 \mathrm{~ms}$; i.e., none of the nucleated bubbles were observed to recondense [49].

Figures 3(c) and 3(d) show a total of 217 individual nucleation events for a driving frequency of $19.6 \mathrm{MHz}$ and 120 individual nucleation events for a frequency of 45.4 MHz, represented as the droplet radius versus the time at which nucleation occurs. The number of droplets that nucleated is shown in the red histogram. The normalized PNP of the driving pulse that results from the superposition of waves transmitted by the 60 electrode pairs is shown in the top panels of Figs. 3(c) and 3(d). Since the electrode pairs are spaced by 1 wavelength, the typical surface wave has a triangular shape originating from 50 superimposed waves. The pressure profile was experimentally verified by optical hydrophone recordings with one end of the $\mathrm{LiNbO}_{3}$ substrate submerged in water. The bubbles are thus more likely to nucleate at the maximum PNP, namely, at the end of the 50 cycle IDT driving pulse. Note the presence of a second event of vaporizations in Fig. 3(d) where a small number of droplets nucleated after the primary wave had passed, most probably a result of an internal reflection in the IDT device. Also, the absence of structure in the data presented in Figs. 3(c) and 3(d) suggests that there is no size dependency on the timing of the vaporization.

The proportion of droplets activated by the ultrasound wave with respect to the total number of droplets present in each separate bin is shown in the histograms displayed in Figs. 4(a) and 4(b) for the driving frequencies of 19.6 and 45.4 MHz, respectively. The resonance curves calculated from the proposed theory are displayed in Figs. 4(c) and 4(d) (red lines). The theoretical resonance peaks in Fig. 4(c) and 4(d) are corrected for the presence of a rigid wall and closely match the experimental peaks in Figs. 4(a) and 4(b).

Numerical simulations were performed on the basis of the geometry of Fig. 2(b) to provide further insight in the
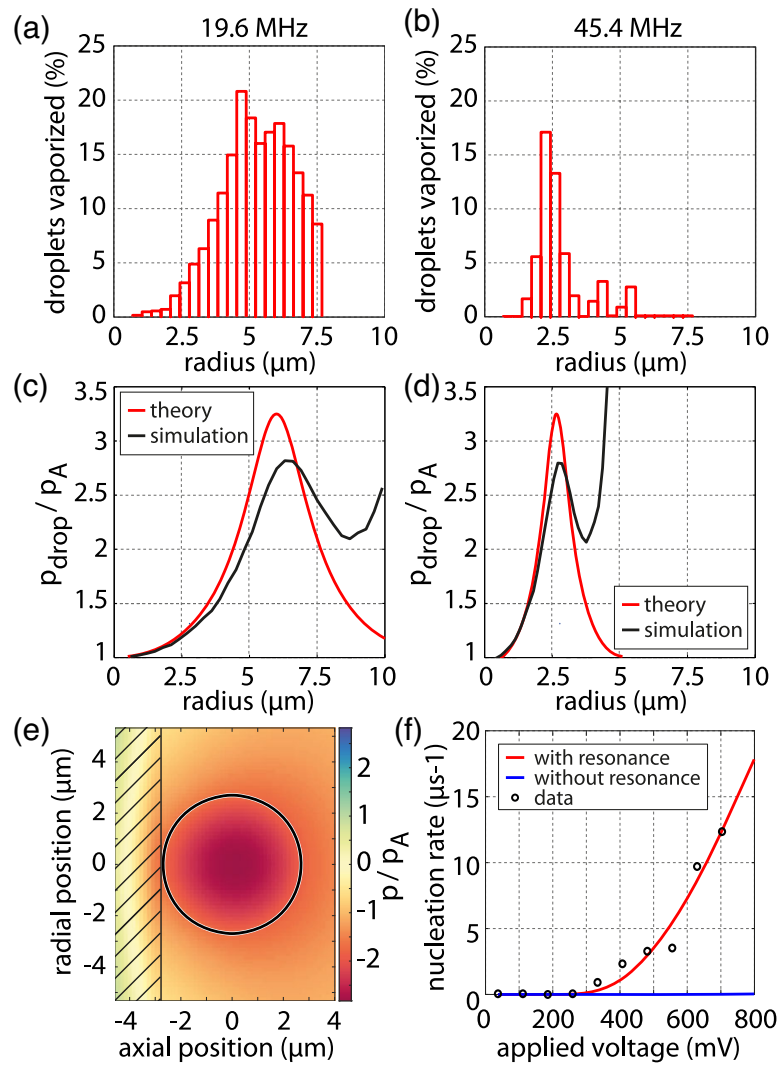

FIG. 4. Resonant vaporization detected at a frequency of 19.6 (a) and $45.4 \mathrm{MHz}$ (b). Calculated and simulated size-dependent pressure amplification factor within the droplet at a frequency of 19.6 (c) and $45.4 \mathrm{MHz}$ (d). (e) Snapshot of the simulated pressure field for a $2.7 \mu \mathrm{m}$ radius droplet resonant at a frequency of 45.4 MHz. (f) Droplet activation probability with and without the resonance effect, where "data" represents the experimental droplet activation probability. 
resonance behavior. The simulations were axisymmetric and computed on a GPU using $k$-wave, an open source MATLAB toolbox for time domain ultrasound simulations in complex media [50], see Supplemental Material SI.5 [36]. The grid size was $0.30 \mu \mathrm{m}$ at a frequency of $19.6 \mathrm{MHz}$ and $0.15 \mu \mathrm{m}$ at a frequency of $45.4 \mathrm{MHz}$. The results are plotted in Figs. 4(c) and 4(d) (black lines). The resulting pressure field in and around the droplet is displayed in the snapshot of Fig. 4(e), taken at $t=375$ ns, i.e., after 17 cycles of ultrasound, see also the Supplemental Material video [36]. The amplification factor of the first resonance, as well as its location, is in very good agreement with the theoretical model, although the simulated amplification is $\sim 15 \%$ lower than predicted by the theory. Interestingly, the presence of a second mode (see SI.5 in Ref. [36]) is visible at $45.4 \mathrm{MHz}$. It appears that the experimental data for a driving frequency of $45.4 \mathrm{MHz}$ [Fig. 4(b)] indeed displays a secondary nucleation peak around a radius of $5 \mu \mathrm{m}$. This higher mode appears to have a smaller effect on the ADV threshold despite the large pressure amplification (factor 10) found in simulation. This is most likely a limitation of the present rigid numerical simulation since higher order nonaxisymmetric modes can induce droplet deformation, which may have a significant impact on the pressure distribution within the droplet.

The statistical increase in the number of vaporization events as a function of the negative acoustic pressure amplitude was investigated by varying the amplitude of the 50-cycle ultrasound pulses at a frequency of $19.6 \mathrm{MHz}$. The envelope of the pressure wave, depicted in the top panels of Figs. 3(c) and 3(d), combined with the measured timing of the event, were used to determine the voltage at which each droplet vaporized. The resulting nucleation rate is displayed in Fig. 4(f) (open circles). Classical nucleation theory [51,52] dictates that the nucleation rate is a function of the surface tension of the liquid $\sigma_{\mathrm{PFP}} \simeq 10 \mathrm{mN} / \mathrm{m}$ [42], of the ambient temperature $T_{\mathrm{amb}}=293 \mathrm{~K}$ and of the ambient pressure:

$\Gamma \propto e^{\left(-\frac{16 \pi}{3 k_{B} T_{\mathrm{amb}}} \frac{\sigma_{\mathrm{PFP}}^{3}}{\left[p_{v}-p_{\mathrm{atm}}-p_{\mathrm{drop}} \cos (\omega t)\right]^{2}}\right)}$

Here, $p_{v}$ is the vapor pressure of the liquid and $k_{B}$ is the Boltzmann constant. The vapor pressure of PFP can be estimated using Antoine's law and ranges from $65 \mathrm{kPa}$ at a temperature of $15^{\circ} \mathrm{C}$ to $95 \mathrm{kPa}$ at $25^{\circ} \mathrm{C}$. As a result, and considering the large peak negative pressures typically required to induce cavitation, $\left(p_{v}-p_{\text {atm }}\right) / p_{\text {drop }} \ll 1$. With this simplification, Eq. (14) can be volume integrated during the negative pressure phase of the ultrasound cycle to determine the average nucleation rate:

$$
\langle\Gamma\rangle \propto \int_{0}^{R_{0}} r^{2}\left[1-\operatorname{erf}\left(\frac{1}{p(r)} \sqrt{\frac{16 \pi \sigma_{\mathrm{PFP}}^{3}}{3 k_{B} T_{\mathrm{amb}}}}\right)\right] d r ;
$$

see details provided in Supplemental Material SI.6 [36]. At resonance the pressure amplification factor due to the resonance effect $p_{\text {drop }} / p_{A} \simeq 3$, see Figs. 4(c) and 4(d). When the SAW devices are driven within their linear range, the acoustic pressure is proportional to the driving voltage. Since the droplet concentration is contained in the prefactor, this expression can be used to fit the experimental data and provide an estimate of the local acoustic driving pressure, which is the only free parameter in Eq. (15). The least-squares fit is shown in Fig. 4(f) and gives $p_{A} / V \simeq 40 \mathrm{MPa} / \mathrm{V}$, leading to an acoustic pressure at the droplet location of $\sim 20 \mathrm{MPa}$ for an excitation voltage of $500 \mathrm{mV}$. Note that these numbers only constitute a rough estimate since, on the one hand, classical nucleation theory is known to overestimate the peak negative pressures required for cavitation [51] and, on the other hand, the range of data available for fitting is limited. It should also be noted that this pressure is of the same order as those typically used for ADV. Interestingly, nucleation theory as presented in Eq. (15) allows us to predict the importance of the resonance effect by calculating the average nucleation rate without resonance, namely, for $p_{\text {drop }} / p_{A}=1$ (with the same prefactor), see Fig. 4(f) (blue line). Thus, it is clear that a pressure amplification factor of 3 has a dramatic effect on droplet vaporization behavior.

In conclusion, it was shown that efficient vaporization can be achieved by driving phase-change PFP droplets at their fundamental resonance frequency. Good agreement was found between the modeled size-dependent pressure amplification within the droplet and the measured sizedependent vaporization probability. Resonance-induced vaporization is a new phenomenon, with important impact on potential ADV strategies and on the understanding on previous experimental observations. In addition, this work shows the potential of using monodisperse phase-change agents driven by a matching resonance frequency to boost the efficiency of ADV.

We thank James Friend for stimulating discussions. This work was funded by NanoNextNL, a micro and nanotechnology consortium of the Government of the Netherlands and 130 partners.

*These authors contributed equally to this work. †.versluis@utwente.nl

[1] M. Delgado, A. Lázaro, J. Mazo, and B. Zalba, Renew. Sustain. Energ. Rev. 16, 253 (2012).

[2] S. Zhang, Y. Shen, L. Wang, J. Chen, and Y. Lu, Appl. Energ. 239, 876 (2019).

[3] J. Alongi, Z. Han, and S. Bourbigot, Prog. Polym. Sci. 51, 28 (2015).

[4] K. E. Wilson, T. Y. Wang, and J. K. Willmann, J. Nucl. Med. 54, 1851 (2013).

[5] P. S. Sheeran and P. A. Dayton, Curr. Pharm. Des. 18, 2152 (2012). 
[6] V. Vinogradov, P. Pavlov, and V. Baidakov, J. Chem. Phys. 128, 234508 (2008).

[7] J. D. Dove, P. A. Mountford, T. W. Murray, and M. A. Borden, Biomed. Opt. Express 5, 4417 (2014).

[8] G. Lajoinie, M. Visscher, E. Blazejewski, G. Veldhuis, and M. Versluis, Photoacoustics 19, 100185 (2020).

[9] O. D. Kripfgans, J. B. Fowlkes, D. L. Miller, O. P. Eldevik, and P. L. Carson, Ultrasound Med. Biol. 26, 1177 (2000).

[10] M. Greenspan and C. E. Tschiegg, J. Res. Natl. Bur. Stand., Sect. C 71C, 299 (1967).

[11] B. Carlier, S. V. Heymans, S. Nooijens, Y. Toumia, M. Ingram, G. Paradossi, E. d'Agostino, U. Himmelreich, J. D'hooge, K. Van Den Abeele et al., Phys. Med. Biol. 65, 065013 (2020).

[12] R. Williams, C. Wright, E. Cherin, N. Reznik, M. Lee, I. Gorelikov, F. S. Foster, N. Matsuura, and P. N. Burns, Ultrasound Med. Biol. 39, 475 (2013).

[13] O. Couture, P. D. Bevan, E. Cherin, K. Cheung, P. N. Burns, and F. S. Foster, Ultrasound Med. Biol. 32, 73 (2006).

[14] P. Sheeran, S. Luois, L. Mullin, T. Matsunaga, and P. A. Dayton, Biomaterials 33, 3262 (2012).

[15] N. Reznik, M. Seo, R. Williams, E. Bolewska-Pedyczak, M. Lee, N. Matsuura, J. Gariepy, F. S. Foster, and P. N. Burns, Phys. Med. Biol. 57, 7205 (2012).

[16] N. Y. Rapoport, Z. Gao, and A. Kennedy, J. Natl. Cancer Inst. 99, 1095 (2007).

[17] P. Mohan and N. Rapoport, Mol. Pharm. 7, 1959 (2010).

[18] S. M. Fix, A. Novell, Y. Yun, P. A. Dayton, and C. B. Arena, J. Ther. Ultrasound 5, 7 (2017).

[19] M. N. Adan, M. L. Fabiilli, C. G. Wilson, and O. D. Kripfgans, in 2012 IEEE Int. Ultrason. Symp. Proc. (IEEE Press, Piscataway, 2012), pp. 448-450.

[20] M. L. Fabiilli, K. J. Haworth, I. E. Sebastian, O. D. Kripfgans, P. L. Carson, and J. B. Fowlkes, Ultrasound Med. Biol. 36, 1364 (2010).

[21] T. Giesecke and K. Hynynen, Ultrasound Med. Biol. 29, 1359 (2003).

[22] K. C. Schad and K. Hynynen, Phys. Med. Biol. 55, 4933 (2010).

[23] M. Aliabouzar, K. N. Kumar, and K. Sarkar, J. Acoust. Soc. Am. 145, 1105 (2019).

[24] W. Kao, S. Kang, and C. Yeh, in 2014 IEEE Int. Ultrason. Symp. Proc. (IEEE Press, Piscataway, 2014), pp. 1790-1793.

[25] J. D. Rojas, M. A. Borden, and P. A. Dayton, Ultrasound Med. Biol. 45, 968 (2019).

[26] S. Lin, G. Zhang, C. H. Leow, and M.-X. Tang, Phys. Med. Biol. 62, 6884 (2017).

[27] X. Lu, X. Dong, S. Natla, O. D. Kripfgans, J. B. Fowlkes, X. Wang, R. Franceschi, A. J. Putnam, and M. L. Fabiilli, Ultrasound Med. Biol. 45, 2471 (2019).

[28] R. Chattaraj, G. Goldscheitter, A. Yildirim, and A. P. Goodwin, RSC Adv. 6, 111318 (2016).

[29] S. Capece, F. Domenici, F. Brasili, L. Oddo, B. Cerroni, A. Bedini, F. Bordi, E. Chiessi, and G. Paradossi, Phys. Chem. Chem. Phys. 18, 8378 (2016).
[30] G. Zhang, S. Lin, C. H. Leow, K. T. Pang, J. Hernandez-Gil, N. J. Long, R. Eckersley, T. Matsunaga, and M.-X. Tang, Ultrasound Med. Biol. 45, 1131 (2019).

[31] S. Raut, M. Khairalseed, A. Honari, S. R. Sirsi, and K. Hoyt, J. Acoust. Soc. Am. 145, 3457 (2019).

[32] O. Shpak, M. Verweij, H. J. Vos, N. de Jong, D. Lohse, and M. Versluis, Proc. Natl. Acad. Sci. U.S.A. 111, 1697 (2014).

[33] C. J. Miles, C. R. Doering, and O. D. Kripfgans, J. Appl. Phys. 120, 034903 (2016).

[34] C. Miles, C. Doering, and O. D. Kripfgans, J. Acoust. Soc. Am. 143, 1835 (2018).

[35] Z. Gol'dberg, Sov. Phys. Acoust. 3, 340 (1957).

[36] See Supplemental Material at http://link.aps.org/supplemental/ 10.1103/PhysRevLett.126.034501 for (I) Full derivation of the model, (II) discussion on the effect of the wall, (III) summary of the material properties used, (IV) note on superharmonic focusing, (V) details and discussion on the numerical simulations and (VI) derivation of the average nucleation rate.

[37] T. G. Leighton, The Acoustic Bubble (Academic Press, New York, 1994).

[38] A. Prosperetti and A. Lezzi, J. Fluid Mech. 168, 457 (1986).

[39] P. Marmottant, M. Versluis, N. de Jong, S. Hilgenfeldt, and D. Lohse, Exp. Fluids 41, 147 (2006).

[40] National Institute of Standards and Technology, NIST/TRC Web Thermo Tables (WTT) NIST Standard Reference Subscription Database 3-Professional Edition Version 22012-1-Pro (2012 (accessed 2020)), http://wtt-pro.nist.gov.

[41] P. Morgado, C. M. C. Laginhas, J. B. Lewis, C. McCabe, L. F. G. Martins, and E. J. M. Filipe, J. Phys. Chem. B 115, 9130 (2011).

[42] M. A. Kandadai, P. Mohan, G. Lin, A. Butterfield, M. Skliar, and J. J. Magda, Langmuir 26, 4655 (2010).

[43] R. Shilton, M. K. Tan, L. Y. Yeo, and J. R. Friend, J. Appl. Phys. 104, 014910 (2008).

[44] Z. Wang and J. Zhe, Lab Chip 11, 1280 (2011).

[45] L. Y. Yeo and J. R. Friend, Annu. Rev. Fluid Mech. 46, 379 (2014).

[46] C. T. Chin, C. Lancée, J. Borsboom, F. Mastik, M. E. Frijlink, N. de Jong, M. Versluis, and D. Lohse, Rev. Sci. Instrum. 74, 5026 (2003).

[47] E. C. Gelderblom, H. J. Vos, F. Mastik, T. Faez, Y. Luan, T. J. A. Kokhuis, A. F. W. van der Steen, D. Lohse, N. de Jong, and M. Versluis, Rev. Sci. Instrum. 83, 103706 (2012).

[48] O. Shpak, T. Kokhuis, Y. Luan, D. Lohse, N. de Jong, J. B. Fowlkes, M. L. Fabiilli, and M. Versluis, J. Acoust. Soc. Am. 134, 1610 (2013).

[49] O. Shpak, L. Stricker, M. Versluis, and D. Lohse, Phys. Med. Biol. 58, 2523 (2013).

[50] B.E. Treeby and B. T. Cox, J. Biomed. Opt. 15, 021314 (2010).

[51] F. Caupin and E. Herbert, C. R. Phys. 7, 1000 (2006).

[52] S. Karthika, T. K. Radhakrishnan, and P. Kalaichelvi, Cryst. Growth Des. 16, 6663 (2016). 